Accelerator Division

Alternating Gradient Synchrotron Department BROOKHAVEN NATIONAL LABORATORY

Upton, New York 11973

Accelerator Division

Technical Note

AGS/AD/Tech. Note No. 426

\title{
MODELING OF MICRO-BUNCHING DURING \\ AGS SLOW EXTRACTION
}

J.W. Glenn

February 20, 1996 


\section{Modeling of Micro-Bunching During AGS Slow Extraction}

J. W. Glenn

\section{Introduction.}

The AGS started extracting micro-bunches in 1974 for Fainberg and Kalogerapoulos to measure anti-neutrons. Bunches were $<\sim 4$ ns spaced every $220 \mathrm{~ns}$. This technique kept the beam bunched in the main RF buckets and moved only the edge of the bunch into the extraction transverse resonance [1].

In $1980 \mathrm{Ch}$ Steinbach and R Cappi used the $200 \mathrm{MHZ}$ SPS prebunching cavity in the PS to reduce the low frequency $(<1 \mathrm{KHz})$ ripple on the PS spill. This method forces the debunched beam between empty buckets, that are centered near the radius of the extraction resonance, by lowering the Main Magnet field. RF structure as an undesirable side effect, was expected, not found to cause the users problems, and thus not investigated [2].

Recently the AGS has run tests powering the $93 \mathrm{MHZ}$ VHF dilution cavity during the SEB spill. This Micro-bunching setup was done as at CERN. The first run with Heavy Ions showed $<2.5 \mathrm{nSec}$ bunches and also spill ripple reduction for a surprising four killo-Hertz variation in VHF frequency. Another run with protons for Experiment 787 showed less than $0.6 \mathrm{nSec}$ bunches, over about a hundred Hertz range. A major fraction of these widths may have been due to detector resolution.

Further understanding of these observations required a computer simulation of longitudinal motion of beam in the AGS with this setup. It shows ripple reduction is predicted for large frequency variation of the VHF cavity voltage and bunches shorter than 100 pico-Sec RMS but over a large frequency range which is not consistent with observations. This precipitated a simulation of the associated transverse motion which indicates that bunches still as short as $130 \mathrm{pSec}$ would be seen during extraction but only for a precise frequency match.

\section{Longitudinal Modeling.}

The equation modeled was 


$$
\begin{gathered}
\dot{\varphi}=W_{0}^{2}\left(\sin \varphi-\sin \varphi_{s}\right) \\
{\left[W_{0}^{2}=\frac{e V \eta f_{R F}^{2} 2 \pi}{\beta^{2} E h}\right]}
\end{gathered}
$$

where $\phi \mathrm{s}$ and equivalent $\mathrm{W}_{0}^{2}$ were calculated using equations in Ref 3 . Normal conditions used were:

$\begin{array}{ll}\text { VHF voltage } & 20 \mathrm{~K} . \\ \text { VHF freq. } & 93.11 \mathrm{MHZ} \\ \text { harmonic \# } & 251 \\ \dot{B} / B & 0.002 / \mathrm{Sec} . \\ \text { proton gamma } & 26.31 \\ \text { AGS radius } & 5057.6 \mathrm{in}\end{array}$

Producing buckets with the following:

$\begin{array}{ll}\text { stable phase } & 0.35^{\circ} \\ \text { synchrotron } \mathrm{f} & 235.3 \mathrm{~Hz} \\ \text { half height } & 10 \mathrm{KV} \\ \text { length } & 344^{\circ} \\ \text { gap between } & 16^{\circ} \text { or } 0.48 \mathrm{nsec}\end{array}$

A particle was started well out of the bucket with a large $\dot{\varphi}$, or frequency difference from the bucket center, and tracked using Quick Basic on a 386. Figure 1 shows this motion; due to the small $\dot{B}(\varphi s)$ the trajectories run together. Figure 2 is with 15 times normal $\dot{B} / B$ to better demonstrate this motion. Location of a particle is plotted every 20 micro-sec equivalent (.01 radians of synchrotron oscillation) as a small dot. The approximate location of part of the separatrix around the empty bucket and beyond is sketched in as solid lines]. As the AGS field falls, the particle spirals out since it is not in the stable bucket; it is not accelerated on average. Being above transition energy, increasing radius implies lower frequency and thus lower $\dot{\varphi}$. At a particular radius the transverse tune is $82 / 3$, and the particle is extracted out of the AGS. This frequency is the rotation frequency of a particle at this radius times the harmonic number of the RF system. The $\dot{\varphi}$ where extraction occurs is the difference between the frequency associated with extraction and the RF frequency in radians per sec. This $\dot{\varphi}$ can be adjusted by changing the extraction radius (by changing the AGS tune) or, more easily, RF frequency. Thus as the beam could be set up to extract at any $\dot{\varphi}$, the parameters are recorded for many different $\dot{\varphi} s$ for the first pass through that particular value (the emphasized points on Fig 2 show these "first crossings" for some $\dot{\varphi}$ s or extraction radii). Note: $\dot{\varphi}$ in these figures is "normalized" to the synchrotron frequency of $235 \mathrm{~Hz}$ or $\sim 1475 \mathrm{radians} / \mathrm{Sec}$. per unit and the phase is offset by $\pi / 2$ to better see the gap between buckets. 
Figure 3 shows the "first crossing" (large dots in Figs 1\&2) results for 300 particles evenly spaced phases at the initial $\dot{\varphi}$. The top series of dots is the starting conditions for these particles, the next is the first phi-dot recorded. All those particles that are decelerated [half] immediately go through the first "extraction resonance"; the others after shifting phase to the deceleration side of the sin wave, thus the particles are spread over one of two pi in phase. By the time the ensemble reach the next $\dot{\varphi}$ that is to be recorded, they appear well bunched. The time phase, $\dot{\varphi}$, and ${ }_{\varphi}$, are recorded at the first crossing of each of a series of $\dot{\varphi}$ 's, or momenta, shown as a series of short lines of many dots of slightly different phases at different $\dot{\varphi} \mathrm{s}$ in Fig. 2 . The assumption is that when the beam crossed the momentum (or $\dot{\varphi}$ ) where the transverse resonance exists, the beam will be extracted almost immediately on that crossing. The tracking continues and for a series of frequencies, each representing another possible resonance radius (or VHF frequency), the same data is recorded. Thus the distribution in phase at many possible extraction resonance radii were plotted and saved on disk for further reduction. These distributions for various frequency offsets were averaged and the RMS width calculated with 123. The results are shown in Fig 4. Figs 5 A-D show histograms of particle population versus time in the $93 \mathrm{MHZ} R F$ cycle for four different $\dot{\varphi} \mathrm{s}$.

One startling result is that the beam will be bunched at the 120 psec level over a broad range of frequencies, larger than the actual bucket height. As the amount of spill structure reduction is inversely related to bunch length [Ref 2], the broad range of frequency differences that still have short bunches is consistent with the experimental result that spill structure is reduced over a large frequency range. The other is that bunch widths of 30 pico-seconds RMS are seen if the bucket is centered at a slightly higher frequency than the one associated with the extraction resonance. This is a bunching factor of almost 400 or a one degree of phase burst of beam at RF frequency.

These results do require that the particles are quickly extracted for a particular momentum, for example have zero emittance, but some coherence, in the transverse direction. This assumption is not completely valid. There are two corrections required.

\section{Momentum width.}

The actual transverse tune of particles of a particular momentum are dependent on their transverse oscillation amplitude; this is primarily due to the resonance driving sextupoles. Due to the amplitude distribution in the transverse direction, at any instant there is a distribution of momenta being extracted (of 2 parts in $10^{4}$ ), the extent in phidot is shown on Fig 2. If the phase of the extracted beam is changing with momentum (or $\dot{\varphi}$ ) the bunches will be wider. The only $\dot{\varphi}$ region that this correction is large is just below synchronous frequency where particles are reaching new momenta as they are accelerating along the bottom of the bucket. This is also unfortunately where the bunches appear narrowest. The resultant bunch width as a function of VHF frequency difference is plotted in Fig 6, here the predicted minima is about $130 \mathrm{pSec}$.

Increasing the AGS's chromaticity would reduce the momentum spread for the required tune spread and thus reduce this correction or increase the allowed frequency error. Other (adverse) effects on extraction from this change would have to be investigated, but are beyond the scope of this paper. 


\section{Delay in extraction - Transverse Modeling.}

- There is some difference in the time to extract particles of the same momentum and transverse oscillation amplitude due to difference phases of betatron oscillation as they enter the resonance. This variation in delay will broaden the bunches depending on the local $\dot{\varphi}$.

Transverse behavior was tracked using equations from Ref 4 . They were modified to be Symplectic by M. Blaskowitz.

$$
\begin{gathered}
X_{(n+1)}=\left(X_{n}+\text { eps } * Y_{n}\right)\left(1-6 * E * Y_{n}\right) \\
Y_{(n+1)}=Y_{n}-\text { eps } * X_{(n+1)}+3 * E *\left[X_{(n+1)}^{2}-Y_{n}^{2}\right]
\end{gathered}
$$

Where $\mathrm{X}$ is related to displacement, $\mathrm{Y}$ to angle, epsilon to tune difference from the resonance and $E$ to drive sextupole strength. $E$ is normalized so Xo the size of the stable region is unity.] Again the motion was tracked on a 386 using a Quick Basic code. 120 particles were started well away from the resonance for various phases and a Gaussian amplitude distribution in XY space and tracked for up to 700,000 turns with the tune changing in time to simulate acceleration by the VHF cavity. (Fig 7 shows a particle tracked for all turns without changing the tune, which is equivalent to $1.9 \mathrm{sec}$ of coasting in the AGS. There was no sign of blow-up or damping, showing the Symplectic condition was met.) For each particle, the time to extraction (blow-up), was recorded if it wasn't retrapped. Using 123, the average and RMS spread in delay time was calculated. These trackings were done for various rates of acceleration or rate of tune change. A plot of variation in delay versus $Q$ dot (Fig 8) shows that the product is invariant over the range of interest. (Note that rate of change of tune is proportional to rate of radius change which is proportional to rate of change of frequency slip to the VHF cavity or phi double dot.)

Efficiency was also calculated as the ratio of those that weren't retrapped to all tried. The efficiency calculated for normal operation is a few percent worse than operational experience indicates for normal Q dots.

The RMS spread in the time delay to the beam leaving the AGS times the rate of slip $(\dot{\varphi})$ for the resonance radius causes an added spread in phase, and thus bunch width, of the extracted beam. This result is "RMS added" to RMS width expected from longitudinal modeling. The results are plotted in Fig 9 with the "raw" width for zero emittance beam and corrections for our beams instantaneous momentum spread and for the variation in delay of extraction.

This effect has little impact on bunch width if the resonance and the bucket are synchronized, thus widths of $130 \mathrm{psec}$ are still expected. The frequency precision required to operate at this point is 
about $+/-50 \mathrm{~Hz}$. At larger $\dot{\varphi}^{\prime}$ 's the effect will be large and bunching should disappear. During the tuneup of VHF cavity frequency for Exp. 787, narrow bunches were not seen at frequency differences that resulted in reduced spill structure.

Some of this delay may be an artifact of the initial conditions in the model which were of equal radius in $\mathrm{XY}$ space. A few tests were done with initial starting positions for more equal energy in the sextupole distorted phase space. This reduced the variation in delay or straggling by a factor of three.

The above modeling was also done for an $80 \mathrm{KV}$ VHF RF system, four times the present voltage. Fig $11 \& 12$ show predicted bunch widths. The minimum predicted width with this new system is $80 \mathrm{pSec}$ RMS or $62 \%$ of the width at $20 \mathrm{KV}$, where $50 \%$ would be expected from simple calculations.

\section{Possible AGS Studies.}

With a high resolution bunch width detector, it would be possible to see the bunch width change with varying $R F$ voltage and also the change by varying the chromaticity. The predicted phase shift at frequencies below synchronous could be verified.

\section{References}

1. Barton "Some Comments on AGS Bunch Areas" AGS DIV 74-3 and Fainberg \& Kalogeropoulos "The AGS Beam Structure" EP\&S 74-2 or BNL 18938, 1974.

2. Cappi \& Steinbach "Improvement of the Low Frequency ..." CERN/PS/OP 80-10 \& IEEE NS-28, No 3, June 1981, pp 2806.

3. CERN's ". SELECTION OF FORMULAE .." CERN/MPS-SI/Int DL/70/4, pp30-31.

4. AGSCD-17 "Effects of Sextupoles ...." M Month 
Fig 1

Single Particle Trajectory - Normal Bdot

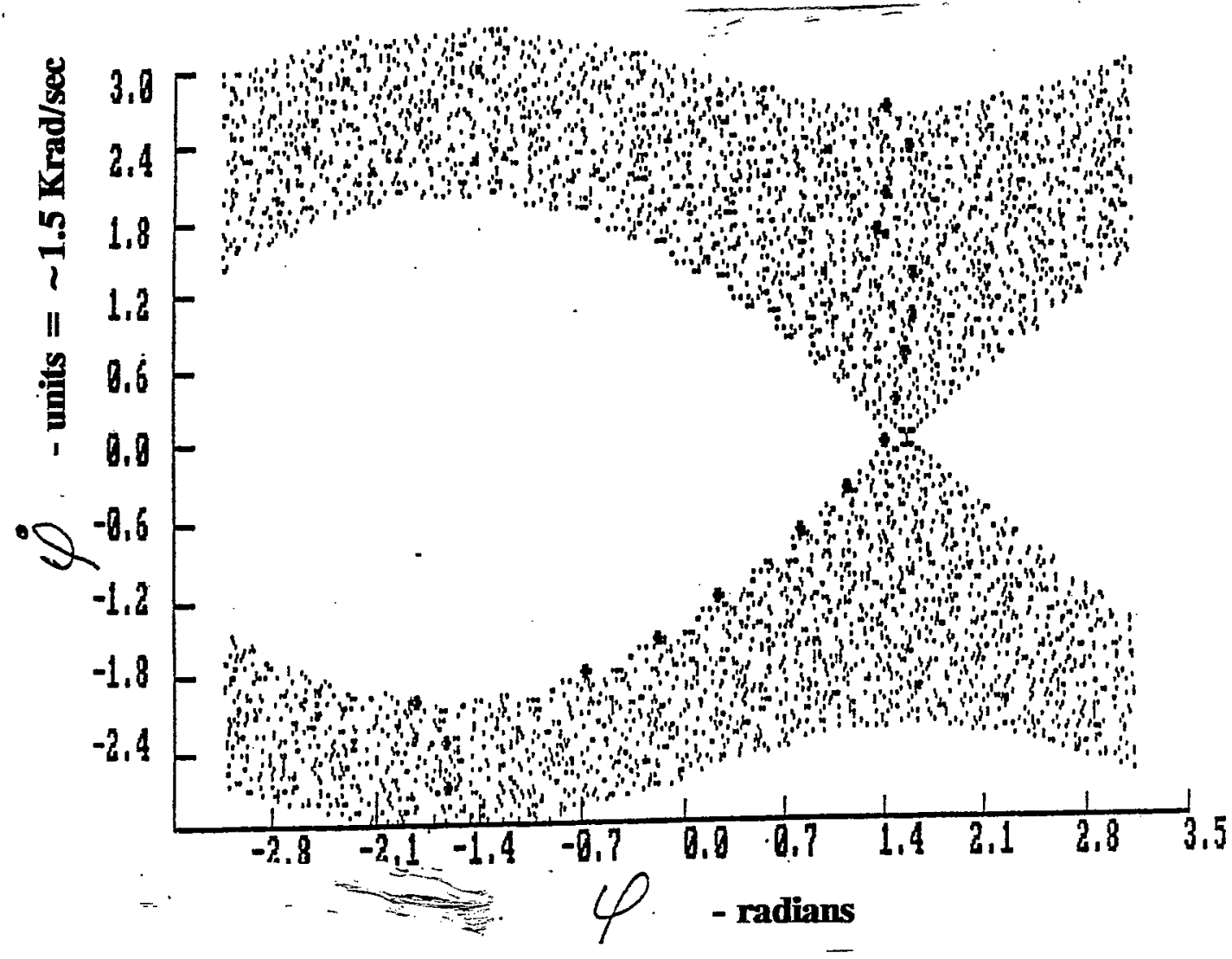

Fig 2

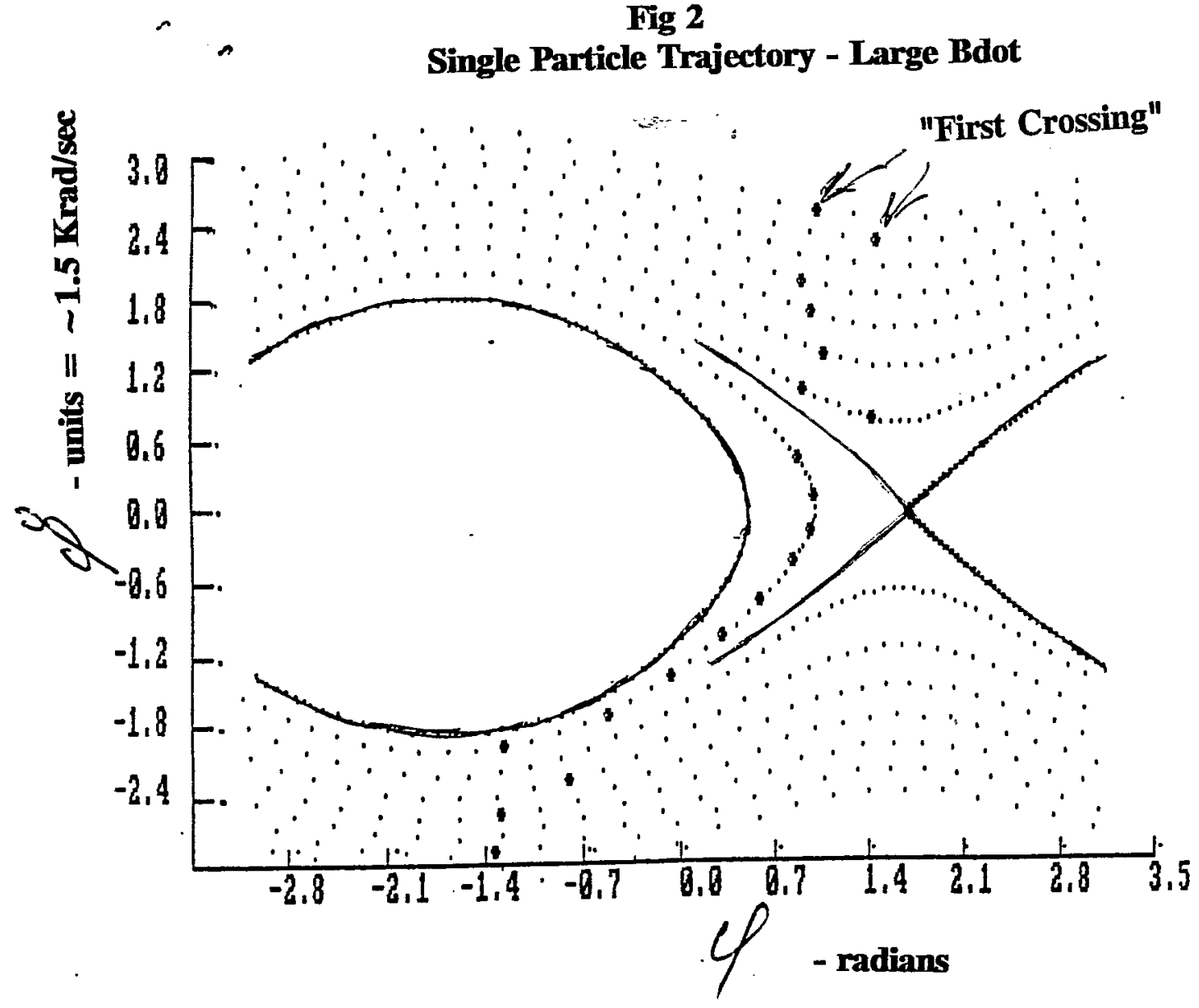




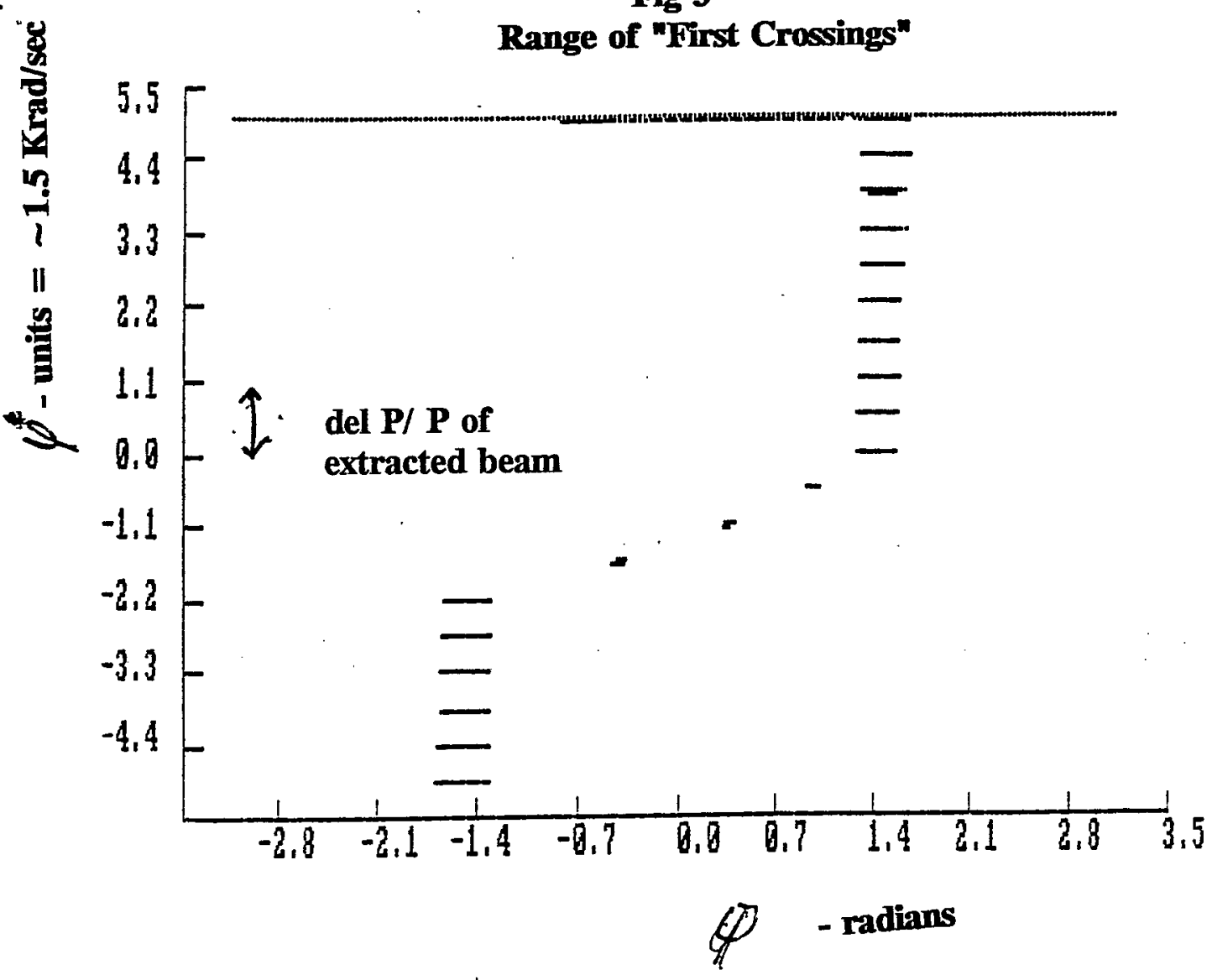

Bunch Width vs Freq Difference

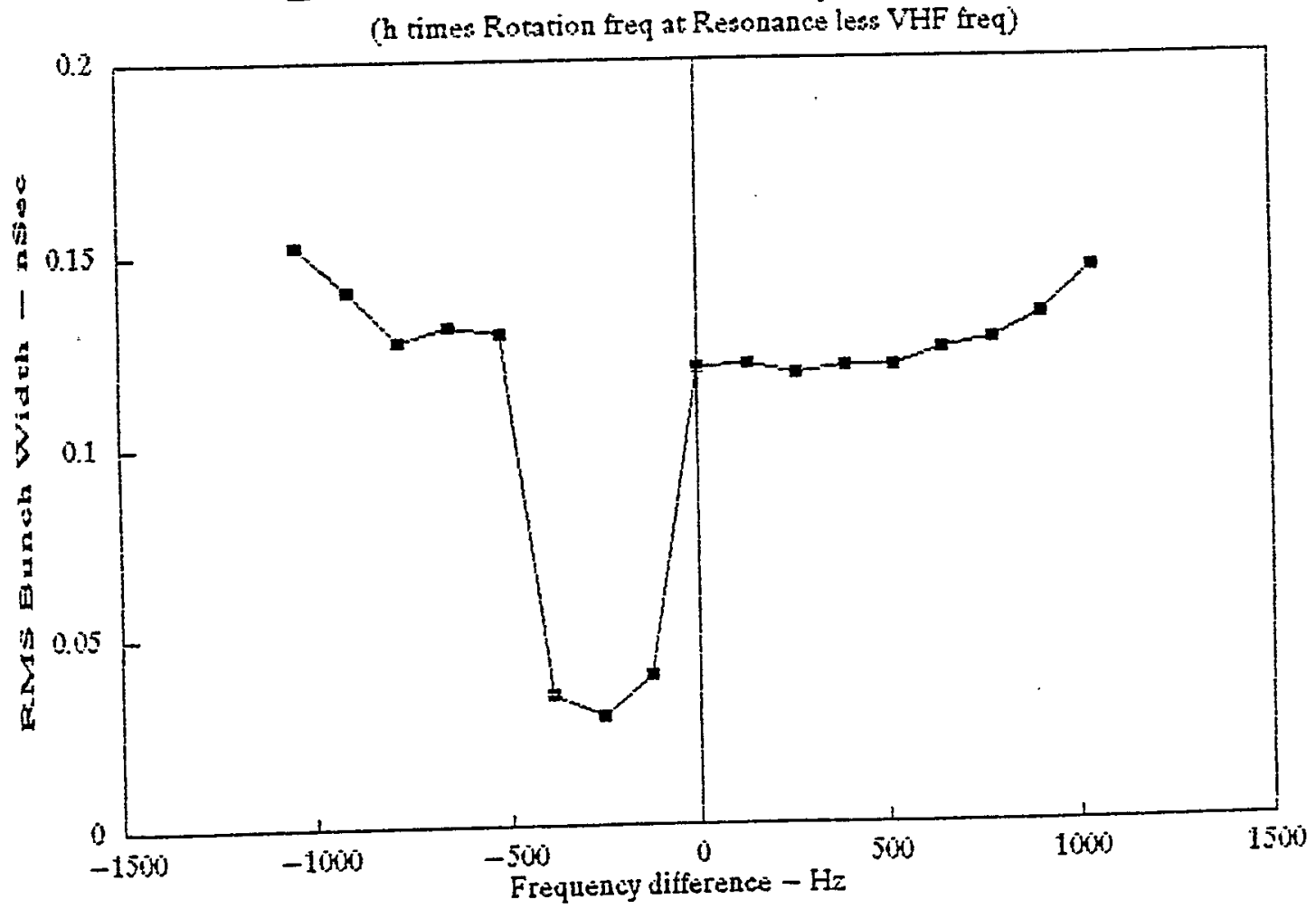


A

UBUNCH DISTRUBRUTION IN TIME

VHF RF req $\sim \mathrm{Hz}$ above resonance

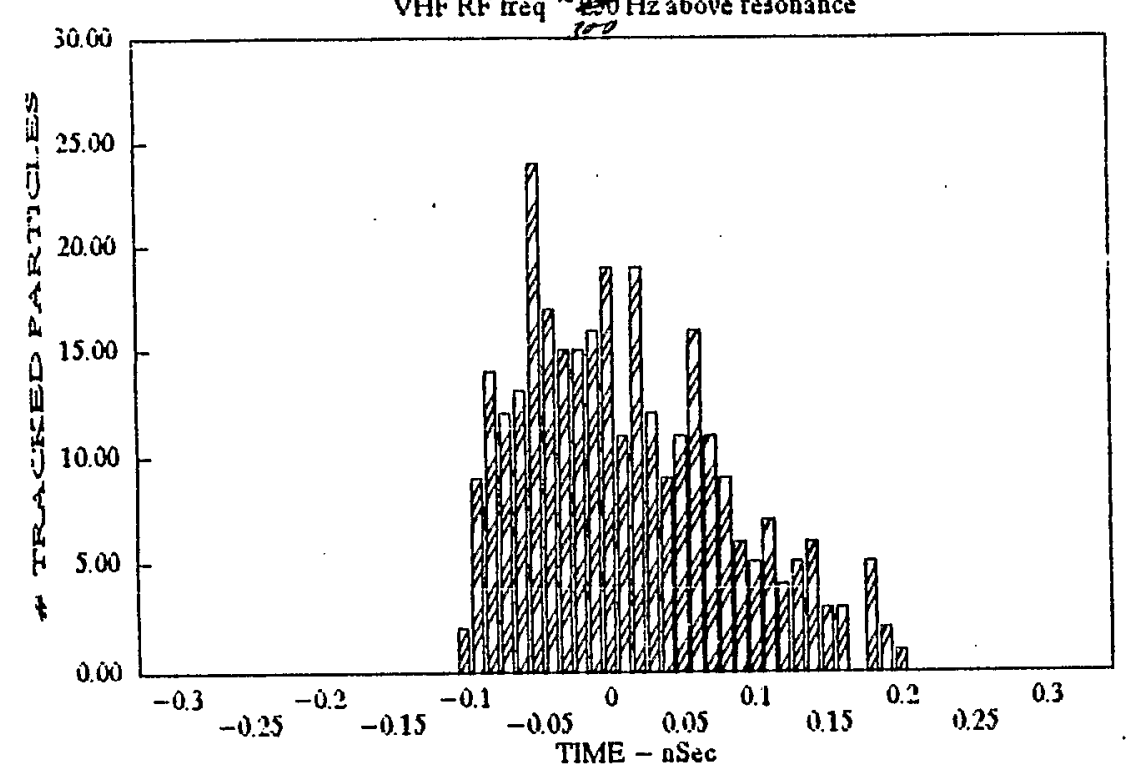

Time Distribution of micro-Bunches

for various frequency offsets
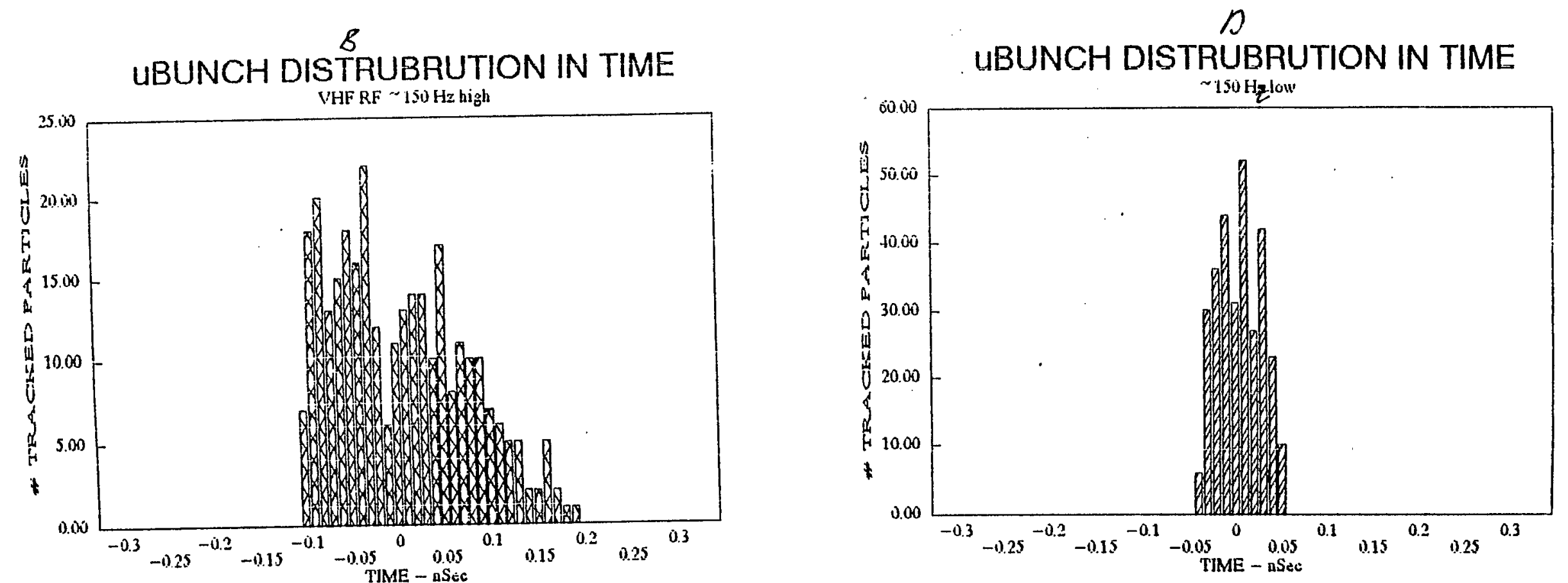
$-9-$

Fig 6

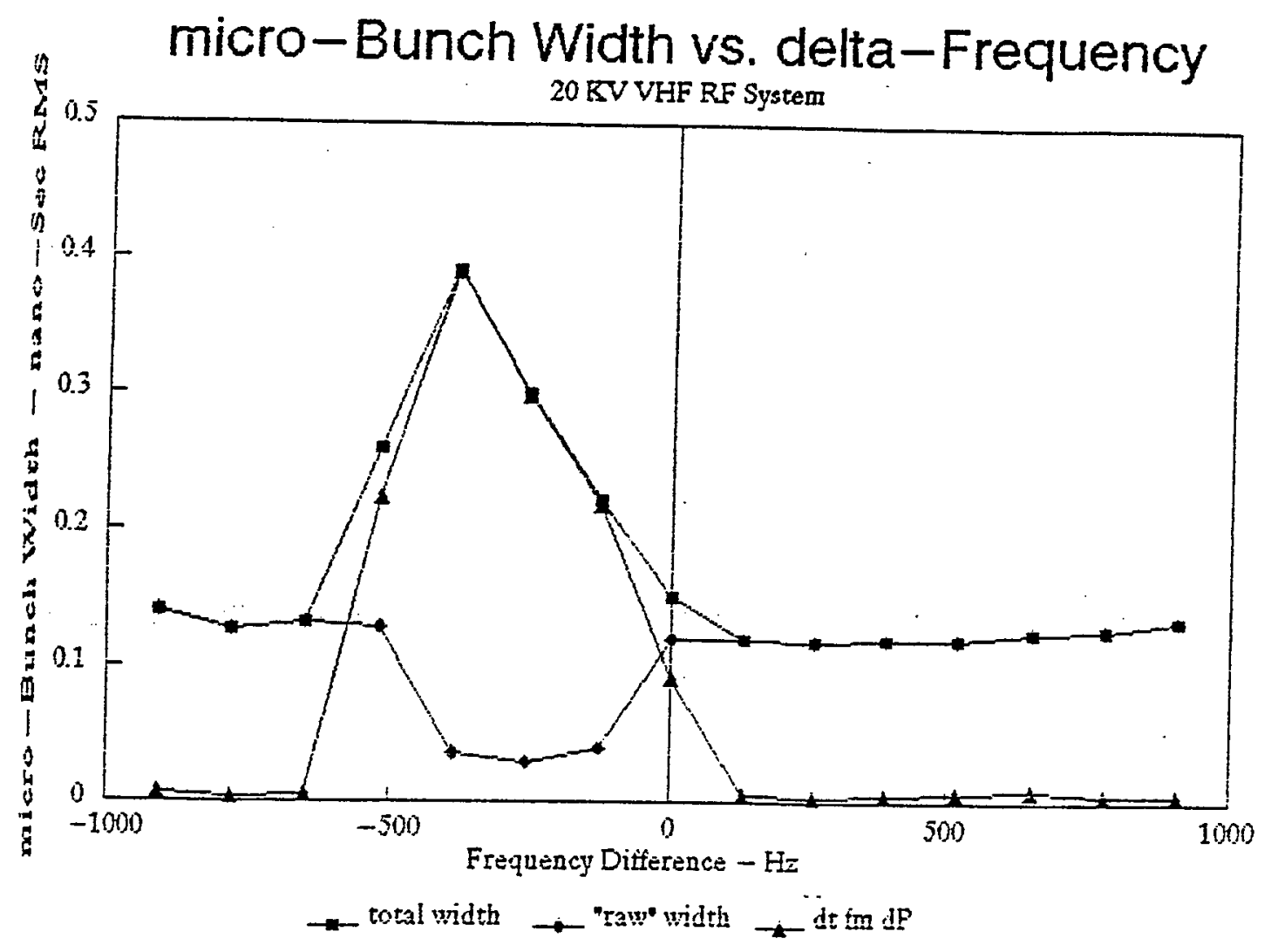

Fig 7

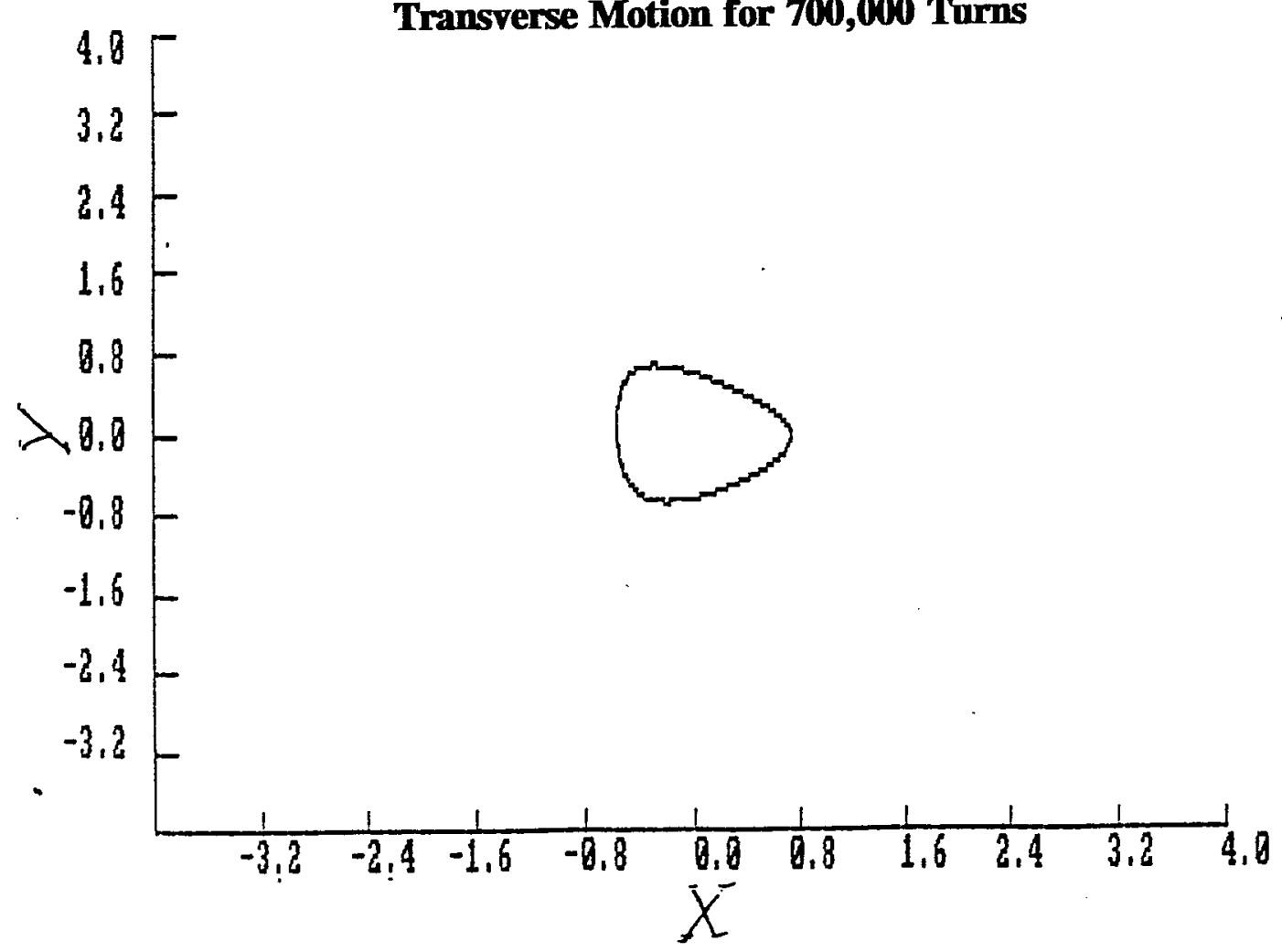




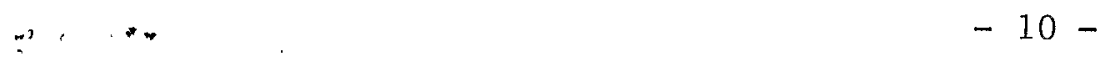

Eig 8

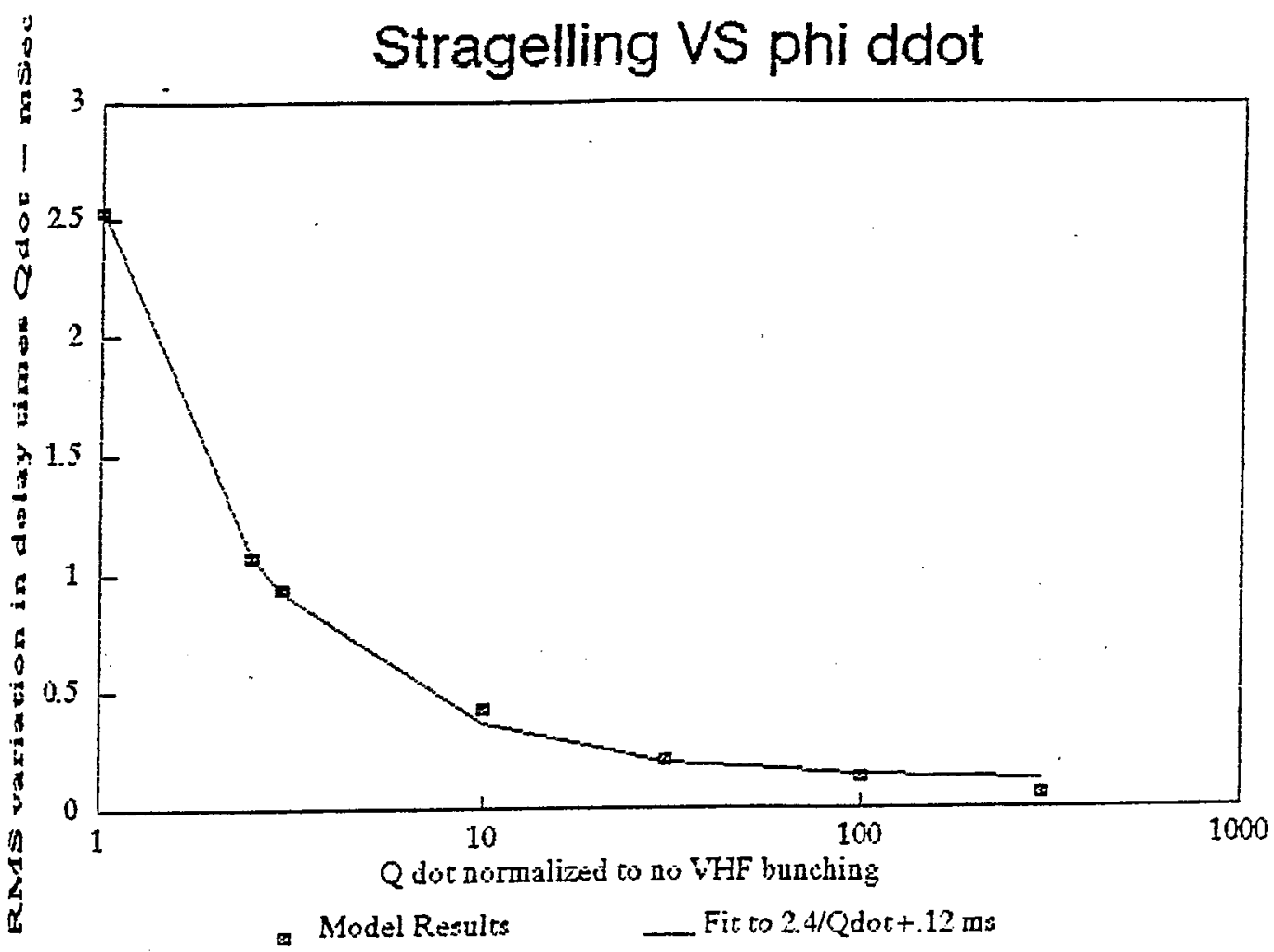

Fig 9

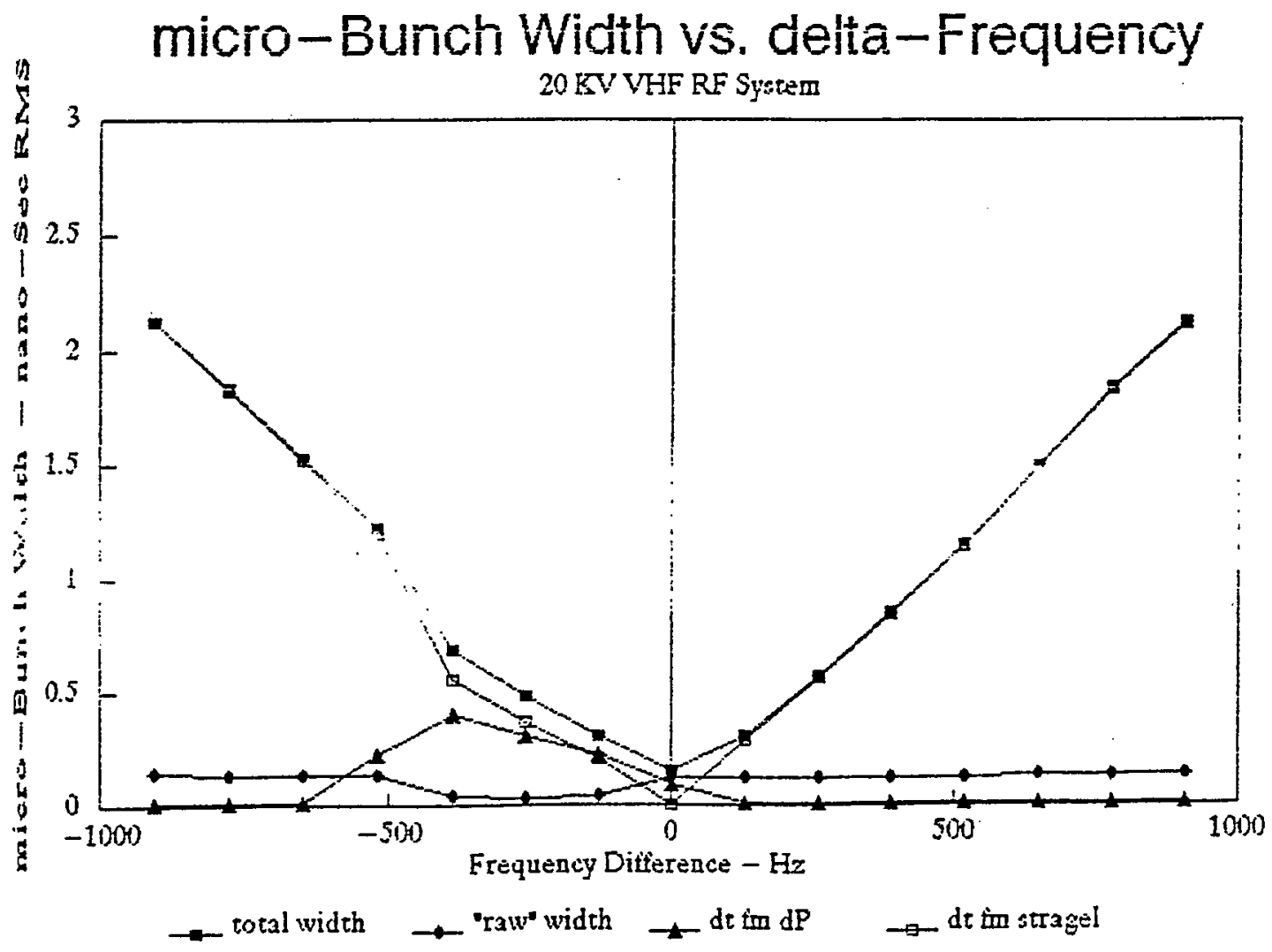


80 KV RF System

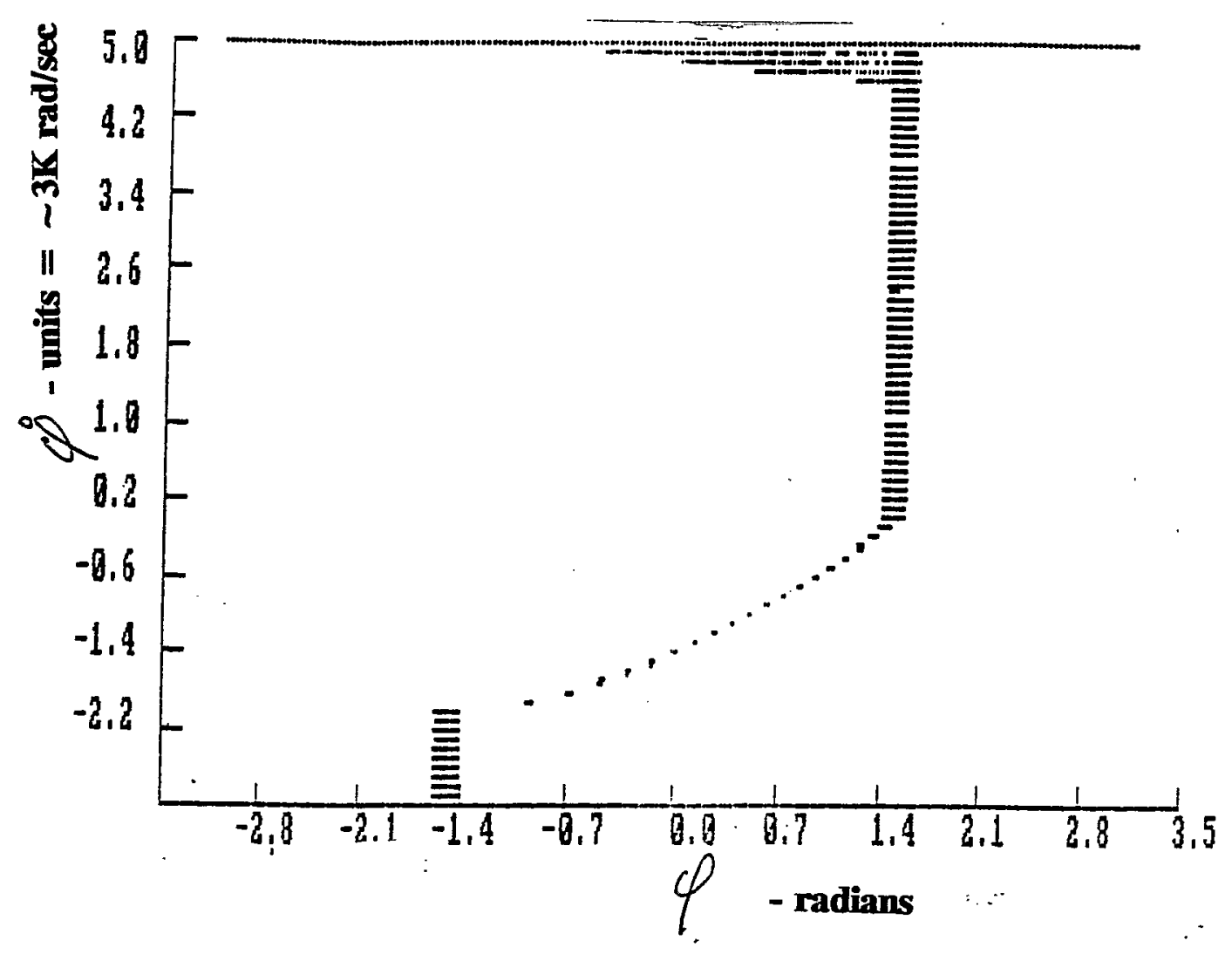

Fig 11

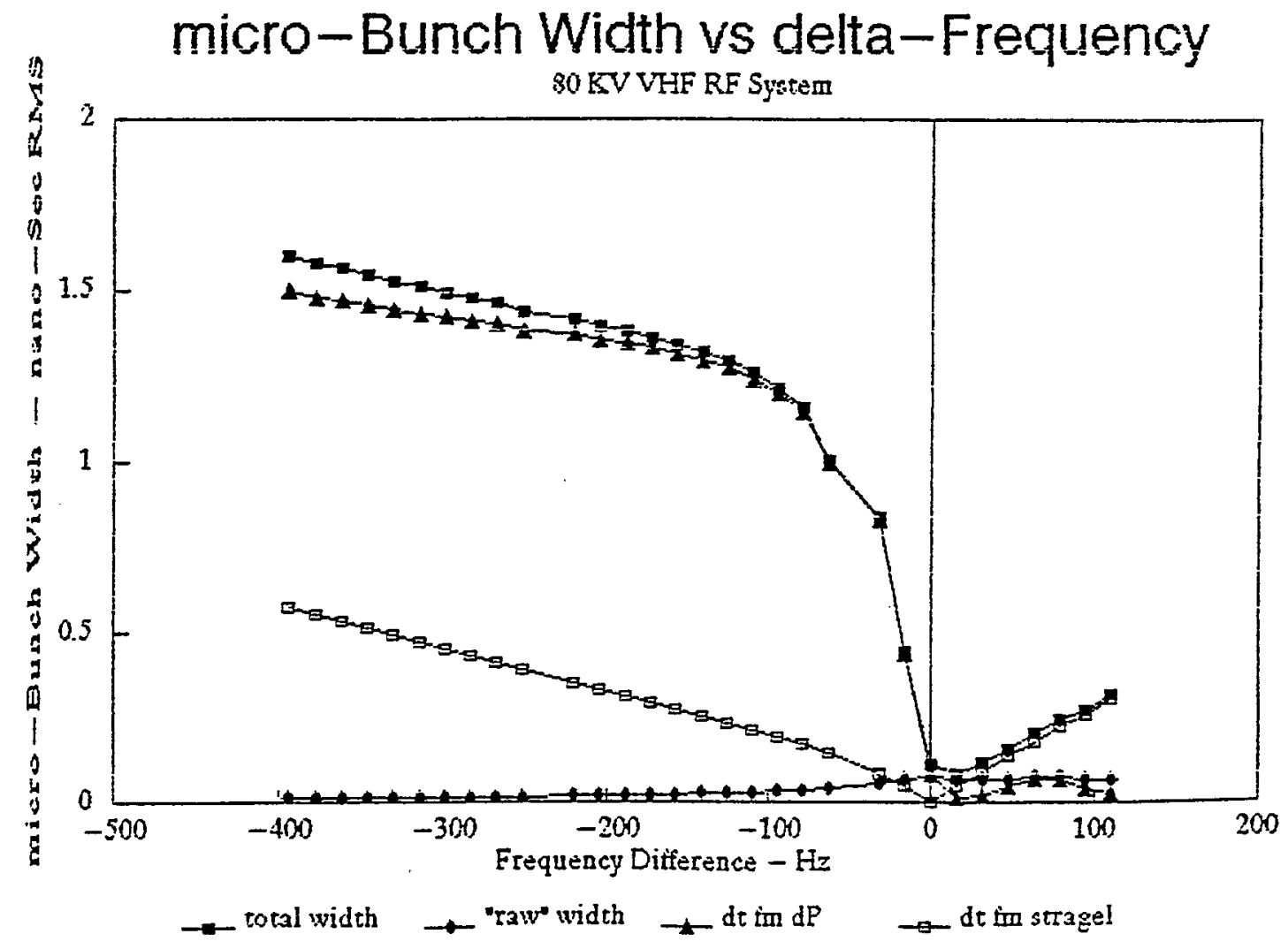

